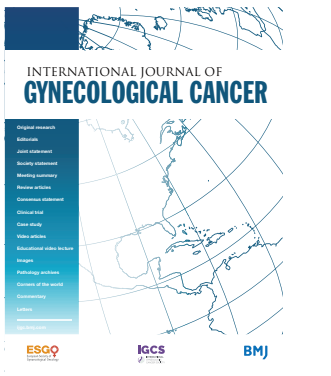

${ }^{1}$ Regional Cancer Treatment Services, MidCentral District Health Board, Palmerston North, New Zealand

${ }^{2}$ Medical Physics, Cancer Diseases Hospital, Lusaka, Zambia

${ }^{3}$ Department of Oncology, Cancer Diseases Hospital, Lusaka, Zambia

${ }^{4}$ Radiation Therapy, Cancer Diseases Hospital, Lusaka, Zambia

Correspondence to Dr Dorothy Lombe, Regional Cancer Treatment Services, MidCentral District Health Board, Palmerston North, New Zealand; dorothylombe@yahoo. com

Received 23 September 2021 Accepted 16 December 2021

Check for updates

(c) IGCS and ESGO 2022. № commercial re-use. See rights and permissions. Published by BMJ.

To cite: Lombe D, M'ule BC, Msadabwe SC, et al. Int J

Gynecol Cancer 2022;32:451456.

\title{
Gynecological radiation oncology in sub- Saharan Africa: status, problems and considerations for the future
}

\author{
Dorothy Lombe (D) , ${ }^{1}$ Barbara Chanda M'ule, ${ }^{2}$ Susan C Msadabwe, ${ }^{3}$ Ernest Chanda ${ }^{4}$
}

\begin{abstract}
Gynecological malignancies in Africa form a significant part of the burden of disease. The high prevalence of HIV in some countries creates a heightened risk for development of human papilloma virus related cancers such as cervical, vulval and vaginal cancers. Radiotherapy is an important modality of treatment for cancer and in Africa compensates for the lack of adequate surgical services for a large proportion of cancers as well as being a cornerstone of treatment for locally advanced cancers and palliation. In this review we look at the status of radiotherapy services in sub-Saharan Africa and critical factors that influence its delivery with a focus on gynecological malignancies. This unveils that radiotherapy for gynecological cancers in sub-Saharan Africa is a significant example of the need for a holistic development approach across different sectors of the economy and different disciplines of medicine. The complexity of its management continues to expose the underdevelopment of health and financial systems and the lack of universal health coverage and social systems as we continue to see unnecessary morbidity and mortality due to the lack of organization. More systematic and scientifically robust investigations tailored to the various sub-Saharan Africa countries need to be conducted to elicit disruptive local solutions to the status quo.
\end{abstract}

\section{INTRODUCTION-THE STATUS}

Radiotherapy is a critical component of cancer treatment, with almost half of patients requiring it at some point in their cancer journey. ${ }^{1}$ In sub-Saharan Africa, where most cancers present at advanced stages, the need is even higher, but the availability of adequate infrastructure and workforce remains an urgent challenge. ${ }^{2}$ The need is compounded by the fact that one of the most common causes of cancer morbidity and mortality is cervical cancer, for which radiotherapy (both external beam and brachytherapy) is an integral part of management.

The Directory of Radiotherapy Centers reports there are a total of 524 radiotherapy machines (420 megavoltage and 104 brachytherapy) in Africa (Figure 1). ${ }^{3}$ The distribution of machines is uneven, with the majority serving mostly the northern and southern parts of the continent. In middle Africa the availability of radiotherapy machines per million people is less than the threshold of 1 at 0.078 , with a grand total of 100 machines (77 megavoltage and 23 brachytherapy). ${ }^{3}$

In this review we attempt to outline the status of radiotherapy for gynecological cancers in subSaharan Africa and factors that contribute to its implementation.

\section{ROLE AND PLACE OF RADIOTHERAPY FOR GYNECOLOGICAL MALIGNANCIES IN SUB- SAHARAN AFRICA}

Cervical cancer is the most common cancer and the standard of care for the management of locally advanced disease includes radiotherapy. ${ }^{45}$ However, other human papilloma virus driven gynecological cancers such as vulva and vaginal cancer are on the rise and radiotherapy has an important role particularly in advanced stage presentation or where there are limited surgical skills. ${ }^{67} \mathrm{~A}$ study in South Africa showed a $16 \%$ annual percentage change (increment) of vulval cancer between 1994 and 2012 with an 18 year median age reduction from 64 to 46 years attributed to the rising HIV prevalence. ${ }^{6}$

Cancer of the corpus uteri in sub-Saharan Africa barely makes it into the top 10 cancers in incidence, at ninth place $(2.2 \%$ of all new female cancer cases in 2020) and is not one of the top 10 causes of female cancer mortality. ${ }^{4}$ These data could be explained partly by a young continental population that may not allow for the natural evolution of the disease before their death, reduced presence of risk factors (eg, obesity, diabetes), poor geriatric services to address those that present with symptoms, and an overwhelmed gynecological oncology surgery service or a lack of such a service. There is a paucity of published literature on endometrial cancers in sub-Saharan Africa, making desk review of the extent of the problem challenging. In addition, cancer registration in sub-Saharan Africa has a multitude of challenges and gaps that are out of the scope of this review. ${ }^{8}$

Ovarian cancer is the fourth most common cause of female cancer morbidity (3.7\%) and fifth for mortality $(4.2 \%)$ on the continent. Generally ovarian cancer carries a high risk of late presentation and poor outcomes, but this risk is worse in Africa where diagnostic and treatment capacity is limited. ${ }^{910}$ In modern 


\section{Review}

\section{Status of Radiation Therapy Equipment}

32236

Countries RT Centres
420

MV Therapy

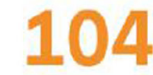

Brachytherapy

Equipment type

(Updated on : 6/23/2021 9:19:53 AM)

MV Therapy
Brachytherapy
Equipment per income groups
(Updated on : 6/23/2021 9:19:53 AM)
High income (H) $\mid 6$
Upper middle income (UM)
Lower middle income (LM)
Low income (L) $\mid 27$

Figure 1 Status of radiotherapy machines in Africa. Figure from dirac.iaea.org

Equipment per regions

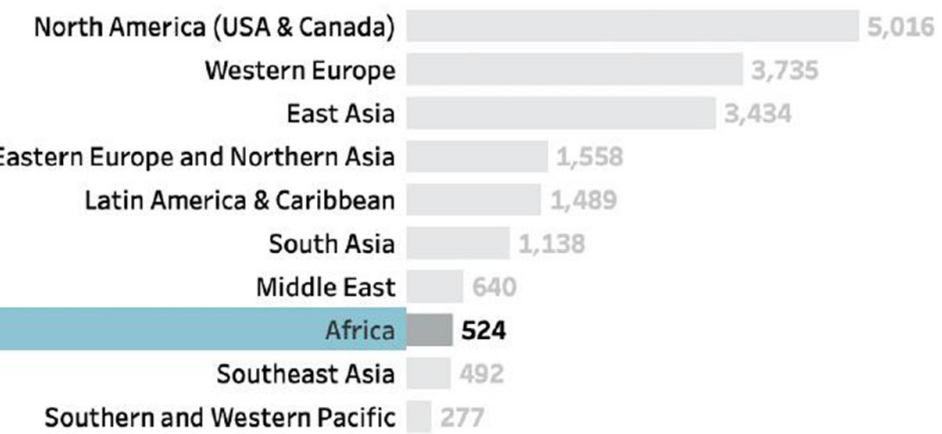

practice, radiotherapy is typically not employed in the management of ovarian cancer, except for localized palliative treatment. ${ }^{11}$

\section{AVAILABILITY AND MAINTENANCE OF EQUIPMENT}

Radiotherapy, a cornerstone in the management of most gynecological malignancies in sub-Saharan Africa, needs to be provided as a safe service and this requires specially constructed infrastructure and expert teams of doctors, medical physicists, radiotherapy technicians and oncology nurses. Cancer management in poorly organized donor driven health systems poses a challenge because acquisition of relevant equipment and building of radiotherapy centers can be discordant with the training and availability of staff. This is due to specific objectives of funders that create silos of service (prevention vs diagnosis vs treatment) without bridges along the continuum of care. ${ }^{12}{ }^{13}$ Consistent access to engineering support for the maintenance of machines and their timely replacement requires appropriate service contracts and procurement regulations, respectively. ${ }^{14}$ Radiotherapy machines are perceived to have a high capital cost, nonetheless this treatment is very effective with larger socio-economic returns for lives saved, particularly in countries where patients present with advanced tumors. ${ }^{15}$ However, the maintenance of machines is concerning for sustainable and consistent cancer control. Elmore and colleagues in their commentary eloquently presented Uganda as a case study on sustainability, highlighting the need for strengthening systems for radiotherapy in Africa. ${ }^{16}$ As machine downtime is inevitable, strong, efficient, reliable machines with robust service contracts must be regarded as unavoidable investments for African countries if gynecological cancer mortality is to be reduced, as an estimated $70 \%$ of patients in low and middle income countries require radiotherapy as part of their treatment plan. ${ }^{14} \mathrm{~A}$ contemporary review of the availability of megavoltage machines in West Africa shows an exponential increase in capacity, particularly in the last decade..$^{17}$ The increase was seen across the region in multiple countries in tandem with the period of an international campaign to increase access to radiotherapy through high level organizations such as the International Atomic Energy Agency, the Union for International Cancer Control and $\mathrm{WHO}^{15}$ 
A sense of satisfaction is created when a country with no previous radiotherapy services acquires at least one machine after years of a lack in services. However, this widens inequality in the same historically disadvantaged countries as those closer to the centers freely access radiotherapy while those who are geographically distant are in an unchanged predicament with little hope of accelerated change. Countries must be cognizant of the false sense of achievement that accompanies singular machine installation, which does not cater for the population burden. An account of availability of radiotherapy services in Nigeria described the first installation of a singular cobalt-60 machine in 1973 followed by a second installation almost a decade and a half later. ${ }^{18}$ The authors reported that for the most populous African country, over a period of 43 years nine radiation therapy centers had been commissioned and at the time of the report only two were functioning at maximal capacity. Important for accountability, two radiotherapy centers had failed to reach their full capacity due to a lack of radiation therapy accessories or the breakdown of essential components, which is a case in point of poor purchase and service contracts familiar to the majority of sub-Saharan African countries.

Unlike some parts of the world that are reconsidering the role of brachytherapy as a necessary part of radiotherapy service delivery, sub-Saharan Africa has no room for such contemplation due to the high prevalence of cervical cancer and a lack of widespread prevention services. In fact, it must look to increasing its high dose rate brachytherapy capacity to meet the ambitious 90-70-90 target of the WHO cervical cancer elimination agenda, which looks at ensuring $90 \%$ of eligible girls are vaccinated against human papilloma virus, $70 \%$ of eligible women are screened for cervical cancer with a high performance test and $90 \%$ of women with invasive cervical cancer have access to quality treatment and palliative care services. ${ }^{19}$ Although the equivalence of low dose rate and high dose rate for cervical cancer treatment has been validated, the volume of patients in sub-Saharan Africa makes a high dose rate a more prudent choice. $^{20}$
Indeed, in a well implemented system, high dose rate brachytherapy can result in an even bigger benefit due to reduced occupancy time of external beam machines providing capacity for other sites such as prostate and penile cancers. A recent policy brief reports only one additional country in sub-Saharan Africa has gained brachytherapy access and 33 countries remain without this service. Therefore, even if there has been a $45 \%$ gain in external beam radiotherapy machine installation, cervical cancer outcomes will remain poor in most of sub-Saharan Africa. ${ }^{21}$

\section{Radiotherapy Workforce}

The radiotherapy human resource needs to compound the continental challenge for quality radiotherapy. An overall deficit in technical experts is persistent due continuous brain drain attributed to a lack of support, recognition and opportunities for growth, particularly for the allied workers in oncology whose critical positions are unfamiliar to the conventional employment system. ${ }^{1}$ A study in 2014 projected that by 2020 there was a need for a human resource expansion of between $102.9 \%$ (radiation oncologists) to $292.3 \%$ (medical physicists) to meet the growing population demands for radiotherapy. ${ }^{2}$ Another study on the oncology workforce showed that the risk of burnout in the already small workforce was high, with African oncologists having a greater workload, lower resources for patients and lower job satisfaction. ${ }^{22}$ The absence of resources to use in daily duties increases moral distress and reduces resilience as evidenced by unpublished results of a survey conducted in Zambia. ${ }^{23}$

The need for an accelerated building of capacity to match the demand is evident, not only for basic knowledge but also for transitioning to more modern techniques and much can be achieved by international collaboration. ${ }^{24}$ Formal education in the radiation sciences improves the safety of radiotherapy delivery and this can be achieved by innovative ways such as blended e-learning and short intensive on-site courses. ${ }^{25} \mathrm{An}$ example of a successful transitioning training program is the Access to Care initiative that trained 13 teams from eight African countries over a period of 5

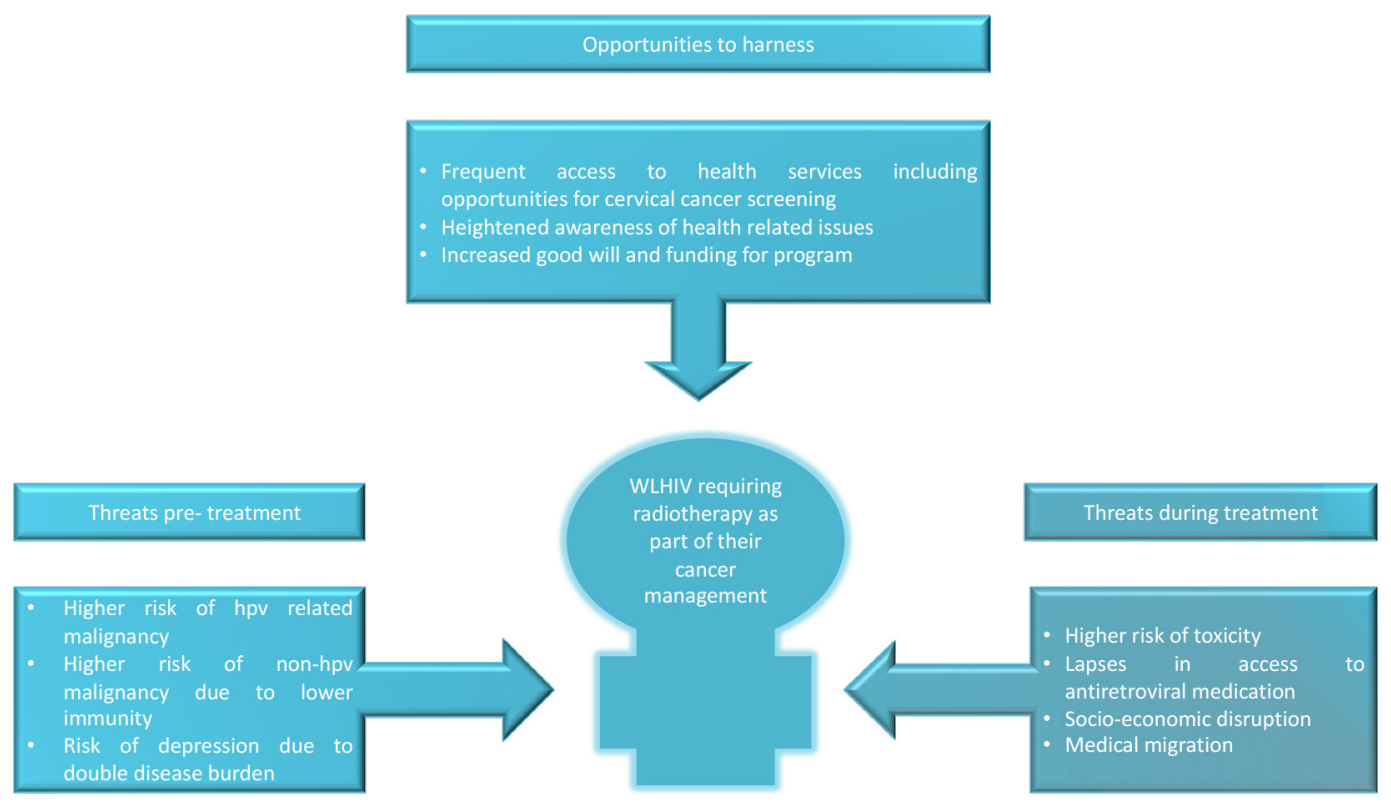

Figure 2 Interaction of factors of women living with HIV and cancer. 


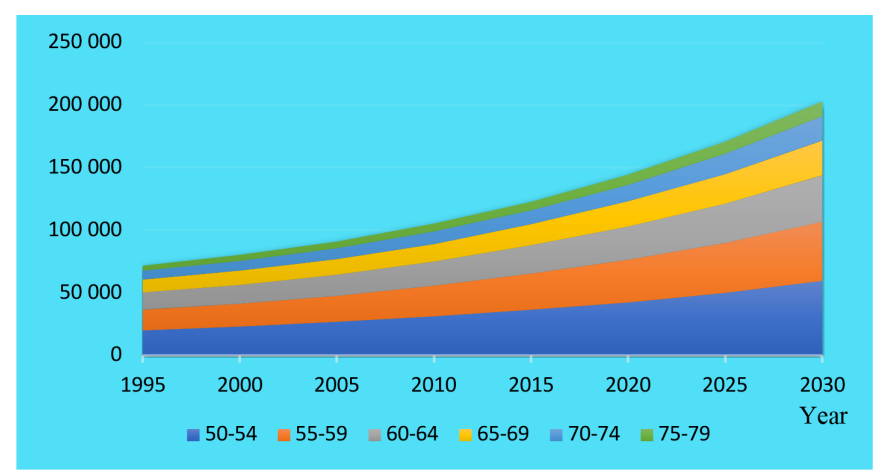

Figure 3 Africa's aging population. Information from population.un.org

years. This initiative was a collaboration between industry and academic institutions that aimed to train professionals intensively on how to implement and maintain three-dimensional techniques in radiotherapy delivery. A commendable aspect of the training program was the holistic approach to the different aspects of radiotherapy access, including radiotherapy department design and procurement processes. As mentioned above, a lack of a robust procurement process and service contracts results in lack of sustainability. ${ }^{24}$

\section{RADIOTHERAPY FOR GYNECOLOGICAL MALIGNANCIES IN WOMEN LIVING WITH HIV IN SUB-SAHARAN AFRICA}

A unique aspect of management of gynecological malignancies in sub-Saharan Africa is the double burden of HIV and cancer (Figure 2). Women living with HIV are at six times higher risk of developing cervical cancer than their seronegative counterparts. ${ }^{26}$ However, multiple factors threaten the success of their treatment. The disease and indeed long-term antiretroviral treatment may compromise their bone marrow health leading to anemia, which is undesirable for external beam radiotherapy. Additionally, the disease and medical therapy for HIV may compromise renal function, presenting a contraindication for cisplatin which contributes significantly to better outcomes.

Long radiotherapy courses prescribed for the radical treatment of gynecological malignancies necessitate the temporary migration of women to centrally placed radiotherapy centers. This migration can lead to a disruption in HIV care. The result is a cascade of negative events such as lack of adherence due to untimely medication refills, leading to development of resistant virus strain mutations and treatment failure. These events create a situation where patients are cured of the cancer but succumb to HIV and opportunistic infections. The current body of literature does not address this problem adequately.

In a report of a prospective cohort of 492 women treated with curative intent for cervical cancer, 5 year overall survival was significantly less in patients who were HIV positive and treated radically at $35.9 \%$ compared with $49 \%(p=0.002)$. ${ }^{27}$ Patients in this study, which can be extrapolated to everyday practice, received HIV care from their local clinics and it was noted that there was limited access to information on adherence to the combined antiretroviral therapy. Newly diagnosed retroviral positive patients were started on combined antiretroviral therapy just before radiotherapy or as soon as possible after starting cancer treatment. The 5 year survival of women living with HIV who were well established on combined antiretroviral therapy was not statistically different compared with that for women who were HIV negative, which shows the importance of appropriate treatment $(49.5 \%$ vs $38.4 \%, p=0.063)$. HIV positive status conferred a poorer outcome even in patients who received adequate doses of radiotherapy. ${ }^{27}$ An early cohort of 259 women from the same group showed that while on curative chemoradiation women living with HIV were more likely to experience hematological toxicity. It was also noted that significantly more women who were HIV positive had chemotherapy omitted from their radical course of treatment. ${ }^{28}$

Comparatively. an updated prospective study of 5 year outcomes of patients with cervical cancer among 142 women living with HIV on long term combined antiretroviral therapy in Botswana showed no difference in survival. ${ }^{29}$ The median cluster of differentiation 4 (CD4) count was 481 cells/ $\mu \mathrm{L}$ and median time on combined antiretroviral therapy was 84 months. In this cohort, after adjusting for HIV status, baseline hemoglobin greater than $10 \mathrm{~g} / \mathrm{dL}$ was a predictor for better outcome and advanced stage a poor prognostic factor.

\section{The Negative Cascade of Uncontrolled Cancer in African Women}

As mentioned above, gynecological malignancies contribute significantly to the burden of cancer in sub-Saharan Africa largely due to cervical cancer. ${ }^{4}$ With an aging population, due to reduced fertility and the positive combined effect of increased early childhood survival and reduced adulthood mortality because of control of communicable diseases, cancer is emerging as an eminent problem (Figure 3). ${ }^{30}{ }^{31}$ Compounding the complexity of low resourced health systems, the female population has historic vulnerabilities on a large scale and women with cancer tend to be secondary priority to other maternal and child health issues in the developing nations' pursuit of attaining Sustainable Development Goals. In spite of their disadvantages and vulnerabilities, sub-Saharan African women form the cornerstone of societal sustainability and development. Their deaths from potentially avoidable causes contribute to the orphan crisis in an environment with insufficient resources to manage this critical social issue.$^{32} \mathrm{~A}$ modeling study defining outcomes of absolute and relative mortality demonstrated that for every 100 women who die from breast and cervical cancer across the African continent, $14-30$ children will also die as a direct association. ${ }^{33}$ The report stated that 'a child's risk of death was derived from the baseline country-specific, all cause mortality rate per 100000 children born multiplied by the excess mortality rate ratio, which is a multiplier of how much higher the rate of dying is for a child whose mother has died compared with a child whose mother is alive in the same population, ${ }^{33}$ Excess mortality rate is defined as the death rate in the general population due to the excess risk imposed by a specific disease ${ }^{34}$ Some observations included that countries with the highest fertility values represented poorer simulation outcomes, underlining the need of a holistic approach to fulfilling the United Nations Sustainable Development Goals.

In another study of women conducted across four African countries (Namibia, Nigeria, Uganda and Zambia), it was estimated that for every 100 deaths from breast cancer (women younger than 50 years), 210 new orphans were created with the number increasing to 247 for Zambia alone. ${ }^{35}$ The study prospectively followed 
1541 women and had $91 \%$ of deaths reported by next of kin who were asked how the death affected the family. The impact of the woman's death included emotional, physical and financial distress. Examples of qualitative information collected were: 'She was the only one who was taking care of her children, so her children may not continue with the education she had wished for them'; 'children were left alone whereby they had to be taken by relatives'; 'she had a family of nine children who were left helpless because she was a single mother'; 'she was a pillar in the family';' 'husband was very sad and worried because he was left with six small children and he didn't know how he would bring them up without the mother'.

Based on these two contemporary studies as illustrations, it is prudent to conclude that taking care of the secondary regarded female population with non-communicable diseases such as cancer will indirectly have a positive effect to achieving the United Nations Sustainable Development Goals.

\section{Future Directions}

A disruptive approach is required to achieve the radiotherapy needs for gynecological malignancies in sub-Saharan Africa. Due to the complexity of procuring robust machines, maintaining the standards of radiotherapy and quality assurance, governments in sub-Saharan Africa must consider ramping up public-private partnership in radiotherapy. The costs of establishing and running radiotherapy services compared with treating infectious diseases and maternal and child health services vary widely. A Nigerian study, although only a small cohort of eight radiation oncologists, showed that the benefits of public-private partnerships in radiotherapy outweighed the potential negative effects. ${ }^{36}$ The participants felt institutions in such an arrangement would probably have improved maintenance of machines, higher patient benefits, larger positive effect on trainees, and increased competition, leading to sustainable radiotherapy services. The main negative effect was the higher costs for patients, which could be mitigated with increased competition.

In conclusion, radiotherapy for gynecological cancers in subSaharan Africa is a significant example of the need for a holistic development approach across different sectors of the economy and different disciplines of medicine. The complexity of its management continues to expose the underdevelopment of health and financial systems as well the lack of universal health coverage and social systems as we continue to see unnecessary morbidity and mortality due to the lack of organization. More systematic and scientifically robust investigations tailored to the various sub-Saharan African countries need to be conducted to elicit disruptive local solutions to the status quo.

\section{Twitter Dorothy Lombe @lombe_dorothy}

Contributors $\mathrm{DL}$ is responsible for the overall content of the manuscript and serves as guarantor for this submission. She initiated the project and participated in the planning, conduct and writing/reporting of the manuscript.BCM participated in the planning, conduct and writing/reporting of the manuscript. SCM participated in the planning, conduct and writing/reporting of the manuscript. EC participated in the planning, conduct and writing/reporting of the manuscript.

Funding The authors have not declared a specific grant for this research from any funding agency in the public, commercial or not-for-profit sectors.

Competing interests None declared.

Patient consent for publication Not applicable.

Ethics approval This study does not involve human participants.
Provenance and peer review Commissioned; internally peer reviewed.

ORCID iD

Dorothy Lombe http://orcid.org/0000-0002-5083-1801

\section{REFERENCES}

1 Atun R, Jaffray DA, Barton MB, et al. Expanding global access to radiotherapy. Lancet Oncol 2015;16:1153-86.

2 Datta NR, Samiei M, Bodis S. Radiation therapy infrastructure and human resources in low- and middle-income countries: present status and projections for 2020. Int J Radiat Oncol Biol Phys 2014;89:448-57.

3 International Atomic Enery Agency. Directory of radiotherapy centres. Available: dirac.iaea.org [Accessed 20 Sep 2021]

4 International Agency for Research on Cancer. Global Cancer Observatory, 2020. Available: https://gco.iarc.fr/today/home [Accessed 5 Sep 2021].

5 Nag S, Erickson B, Thomadsen B, et al. The American brachytherapy Society recommendations for high-dose-rate brachytherapy for carcinoma of the cervix. Int J Radiat Oncol Biol Phys 2000;48:201-11.

6 Chikandiwa A, Pisa PT, Sengayi M, et al. Patterns and trends of HPV-related cancers other than cervix in South Africa from 19942013. Cancer Epidemiol 2019;58:121-9.

7 de Martel C, Plummer M, Vignat J. Worldwide burden of cancer attributable to HPV by site, country and HPV type: worldwide burden of cancer attributable to HPV. Int J Cancer 2017;141:664-70.

8 Omonisi AE, Liu B, Parkin DM. Population-Based cancer registration in sub-Saharan Africa: its role in research and cancer control. JCO Glob Oncol 2020;6:1721-8.

9 Chornokur G, Amankwah EK, Schildkraut JM, et al. Global ovarian cancer health disparities. Gynecol Oncol 2013;129:258-64.

10 Rambau PF, Köbel M, Tilley D, et al. Ovarian cancer: diagnostic accuracy and tumor types distribution in East Africa compared to North America. Diagn Pathol 2020;15:86.

11 Butala AA, Patel RR, Manjunath S, et al. Palliative radiation therapy for metastatic, persistent, or recurrent epithelial ovarian cancer: efficacy in the era of modern technology and targeted agents. Adv Radiat Oncol 2021;6.

12 Abdel-Wahab M, Gondhowiardjo SS, Rosa AA, et al. Global radiotherapy: current status and future Directions-White paper. JCO Glob Oncol 2021;7:827-42.

13 Lombe D, Msadabwe S, Maka M, et al. Zambia cervical cancer control program resource mapping - Leveraging support to achieve the 90-70-90 elimination target. J Cancer Policy 2021;28.

14 Barton MB, Frommer M, Shafiq J. Role of radiotherapy in cancer control in low-income and middle-income countries. Lancet Oncol 2006;7:584-95.

15 Lievens Y, Gospodarowicz M, Grover S, et al. Global impact of radiotherapy in oncology: saving one million lives by 2035. Radiother Oncol 2017;125:175-7.

16 Elmore SN, Sethi RV, Kavuma A, et al. Broken machines or broken systems: the road to meaningful global radiotherapy access. J Glob Oncol 2017;3:438-40.

17 Taku N, Polo A, Zubizarreta EH. External beam radiotherapy in Western Africa: 1969-2019. Clin Oncol 2021.

18 Irabor OC, Nwankwo KC, Adewuyi SA. The Stagnation and decay of radiation oncology resources: lessons from Nigeria. Int J Radiat Oncol Biol Phys 2016;95:1327-33.

19 World Health Organisation. Global strategy to accelerate the elimination of cervical cancer as a public health problem. WHO, 2020.

20 Scott AA, Yarney J, Vanderpuye V, et al. Outcomes of patients with cervical cancer treated with low- or high-dose rate brachytherapy after concurrent chemoradiation. Int J Gynecol Cancer 2021;31:670-8.

21 Elmore SNC, Polo A, Bourque J-M, et al. Radiotherapy resources in Africa: an international atomic energy agency update and analysis of projected needs. Lancet Oncol 2021;22:e391-9.

22 Vanderpuye V, Hammad N, Martei Y, et al. Cancer care workforce in Africa: perspectives from a global survey. Infect Agent Cancer 2019;14:11.

23 Lombe D, M'ule B, et al. Stress, Resilience and Moral Distress among Health Care Providers and Staff at Zambia's national Cancer Diseases Hospital during the COVID-19 Pandemic. Unpublished work.

24 Burger H, Wyrley-Birch B, Joubert N. Bridging the radiotherapy education gap in Africa: lessons learnt from the Cape town access 


\section{Review}

to care training programme over the past 5 years (2015-2019). Journal of Cancer Education 2021;128.

25 Stecklein SR, Taniguchi CM, Melancon AD, et al. Radiation sciences education in Africa: an assessment of current training practices and evaluation of a high-yield course in radiation biology and radiation physics. JCO Glob Oncol 2020;6:1631-8.

26 Stelzle D, Tanaka LF, Lee KK, et al. Estimates of the global burden of cervical cancer associated with HIV. Lancet Glob Health 2021;9:e161-9.

27 Simonds HM, Botha MH, Neugut Al, et al. Five-Year overall survival following chemoradiation among HIV-positive and HIV-negative patients with locally advanced cervical carcinoma in a South African cohort. Gynecol Oncol 2018;151:215-20.

28 Simonds HM, Neugut AI, Jacobson JS. Hiv status and acute hematologic toxicity among patients with cervix cancer undergoing radical chemoradiation. Int $J$ Gynecol Cancer 2015;25:884-90.

29 MacDuffie E, Bvochora-Nsingo M, Chiyapo S, et al. Five-Year overall survival following chemoradiation therapy for locally advanced cervical carcinoma in women living with and without HIV infection in Botswana. Infect Agent Cancer 2021;16:55.
30 United States Census Bureau. Census bureau releases new report on aging in Africa, 2020. Available: https://www.census.gov/ newsroom/press-releases/2020/aging-in-africa [Accessed 5 Sep 2021].

31 United Nations. United nations department of economic and social Affairs population dynamics. published, 2019. Available: https:// population.un.org [Accessed 5 Sep 2021].

32 Lombe M, Ochumbo A. Sub-Saharan Africa's orphan crisis: challenges and opportunities. Int Soc Work 2008;51:682-98.

33 Mailhot Vega RB, Balogun OD, Ishaq OF. Estimating child mortality associated with maternal mortality from breast and cervical cancer: Mother's Cancer Mortality \& Child Death. Cancer 2019;125:109-17.

34 Lenner P. The excess mortality rate. A useful concept in cancer epidemiology. Acta Oncol 1990;29:573-6.

35 Galukande M, Schüz J, Anderson BO, et al. Maternally orphaned children and intergenerational concerns associated with breast cancer deaths among women in sub-Saharan Africa. JAMA Oncol $2021 ; 7$.

36 Anakwenze Akinfenwa CP, Ibraheem A, Nwankwo K, et al. Emerging use of public-private partnerships in public radiotherapy facilities in Nigeria. JCO Glob Oncol 2021;7:1260-9. 